\title{
DISEÑO DE UN AMBIENTE DE APRENDIZAJE MEDIADO POR TIC PARA LA ENSEÑANZA DE OPERADORES MECÁNICOS ORIENTADO AL GRADO SÉPTIMO DE LA EDUCACIÓN BÁSICA, EN EL COLEGIO BOYACÁ DE DUITAMA
}

\author{
DESIGN OF A LEARNING ENVIRONMENT MEDIATED BY ICT FOR THE \\ TEACHING OF MECHANICAL OPERATORS ORIENTED TO THE SEVENTH \\ GRADE OF BASIC EDUCATION, IN THE BOYACÁ SCHOOL OF DUITAMA.
}

\author{
MSc(c). Carmen Emilce Barrera Mesa, PhD. Flavio Humberto Fernández Morales \\ PhD. Julio Enrique Duarte \\ Universidad Pedagógica y Tecnológica de Colombia, Duitama, Boyacá, Colombia \\ Grupo de investigación DECTEN. \\ E-mail:\{emybarreram, flaviofm1, julioenriqued1\}@gmail.com
}

\begin{abstract}
Resumen: En este artículo se presenta el diseño de un ambiente de aprendizaje mediado por las Tecnologías de la Información y la Comunicación (TIC), para la enseñanza de los operadores mecánicos, con el objetivo de mejorar el rendimiento académico en el área de Tecnología e Informática. El ambiente va dirigido a estudiantes del grado séptimo de la educación básica, del Colegio Boyacá de Duitama. Los contenidos se crean a través de Objetos de Aprendizaje (OA), exportados como paquetes SCORM y organizados en la plataforma Moodle, donde el estudiante tiene la oportunidad de participar en foros, chats, realizar tareas y resolver cuestionarios que complementan y evalúan el tema. Como resultado se presenta la estructura del ambiente de aprendizaje, la organización de los contenidos y la estrategia didáctica prevista para su utilización.
\end{abstract}

Palabras clave: Operadores mecánicos, mediación de TIC, ambiente de aprendizaje, enseñanza de tecnología, SCORM.

\begin{abstract}
This paper presents an environments of learning design that has been mediated through the Information and Communication Technology (ICT), in order to learn mechanical Operators, with the aim of getting better academicals results in the field of informatics technology. The students in seventh graders have been involved in order to learn basic education in Boyacá School in Duitama. The subjects were designed trough the objects of learning $(\mathrm{OL})$, have been exported from SCROM, organized in the Moodle platform, where the student has the opportunity in order to participate in forums, chats, doing the homework and answering the questions which complete and evaluate the subject. As a result the structural giving of learning environments, the contents of organization and the didactic a strategy have been presented for the students, utilization.
\end{abstract}

Keywords: Mechanical operators, TIC mediation, learning environment, SCORM.

\section{INTRODUCCIÓN}

En Colombia, el área de Tecnología e Informática se implementó como área fundamental y obligatoria a partir de la promulgación de la ley general de educación, en su artículo 23, y desde entonces, hace parte del currículo de las instituciones educativas (Montes, 2013; Niño et al., 
2016). El Ministerio de Educación Nacional, MEN, en la guía 30: Ser Competente en Tecnología, estableció 4 componentes orientadores para el área, a saber: Naturaleza y Evolución de la Tecnología, Apropiación y uso de la Tecnología, Solución de Problemas con Tecnología, y Tecnología y Sociedad (MEN, 2008). Específicamente, en el primer componente se señala que los estudiantes deben adquirir la competencia para reconocer principios, conceptos y funciones propias de los artefactos, que les permitan dar solución a problemas de su entorno por medio de proyectos tecnológicos (Angarita et al., 2014, Torres Clayton J et al., 2013).

Los operadores mecánicos son parte fundamental en la formación tecnológica según las tendencias de enseñanza de las ciencias, sugeridas por el enfoque Ciencia, Tecnología y Sociedad, CTS (Solbes y Vilches, 1992). Su importancia radica en que el medio que nos rodea está formado por operadores mecánicos y que ellos, junto con los operadores eléctricos, son los componentes principales de los artefactos que usamos a diario (Angarita et al., 2016; González et al., 2016).

Lo anterior hace indispensable que el estudiante conozca el concepto de operadores mecánicos, su principio de funcionamiento y la aplicación de cada uno de ellos, para que con base en ese aprendizaje y conocimiento de su entorno pueda apropiarse y usar la tecnología para solucionar problemas que afecten su comunidad.

La experiencia de los autores en la enseñanza del área de Tecnología e Informática, ha permitido identificar la falta de interés de los estudiantes para lograr los desempeños correspondientes a la parte tecnológica. La principal debilidad se presenta con la identificación, utilidad e implementación de los operadores mecánicos en proyectos tecnológicos.

En el grado séptimo, de acuerdo con la Guía 30, el estudiante debería reconocer, en algunos artefactos: conceptos, funcionamiento y principios científicos que permitieron su creación; además de explicar el concepto de sistema e indicar los componentes y las relaciones de causa y efecto para proponer soluciones tecnológicas a problemas de su entorno. Es decir, el estudiante debe conocer cómo están conformados y cómo funcionan los artefactos que lo rodean para poder plantear soluciones acordes a la necesidad de su contexto.

En el caso de la institución objeto de estudio, el Colegio Boyacá de Duitama, no se cuenta con el aula de tecnología ni con los elementos necesarios para trabajar con artefactos y explorar los operadores mecánicos; sin embargo, tiene dos salas de informática, equipadas cada una con: 40 computadores portátiles, un Subwoofer y un tablero táctil de 60 pulgadas. Para optimizar estas salas, surge la necesidad de crear un Ambiente de Aprendizaje mediado por TIC que permita a los estudiantes adquirir conocimientos de una manera más dinámica y participativa (Ávila A. A. et al., 2012).

Este trabajo presenta el diseño y desarrollo de un ambiente de aprendizaje mediado por TIC, para la enseñanza del tema operadores mecánicos, con el fin de que los estudiantes se apropien de esta temática. Igualmente, se sugiere el diseño didáctico para la implementación del ambiente. De esta manera se espera remediar la falta de espacio físico para el desarrollo de laboratorios prácticos, a la vez que se aprovechan los dispositivos informáticos disponibles en la institución.

\section{MARCO TEÓRICO Y MÉTODOS}

\subsection{Conceptos básicos}

\subsubsection{Ambientes de aprendizaje y TIC}

Jaramillo et al., (2005), plantean que un ambiente de aprendizaje es "Un espacio construido por el profesor con la intención de lograr unos objetivos de aprendizaje concretos, esto significa realizar un proceso reflexivo en el que se atiende a las preguntas del qué, cómo y para qué enseño"(p. 21). De esta manera, un ambiente de aprendizaje permite a los estudiantes obtener herramientas para interactuar y realizar las actividades que los llevarán a alcanzar los propósitos educativos para los que fue construido (Rodríguez, 2016).

Los ambientes de aprendizaje incorporan material didáctico que puede ser real o virtual, dependiendo de las temáticas a trabajar, del nivel de formación y de la infraestructura disponible (Garcés et al., 2014; Castro et al., 2014; Núñez et al., 2014).

Los procesos de enseñanza aprendizaje presentan nuevas metodologías debido a las Tecnologías de la Información y la Comunicación, TIC, que Adell (1997) citado por Boude y Medina (2011) define como "el conjunto de instrumentos y procedimientos que permiten la adquisición, producción, almacenamiento, tratamiento, comunicación, registro y presentación de informaciones, en forma de voz, imágenes y datos contenidos en señales de naturaleza acústica, óptica 
o electromagnética. Las TIC incluyen la electrónica como tecnología base que soporta el desarrollo de las telecomunicaciones, la informática y el audiovisual" (p.303).

Gracias a su versatilidad para la presentación e intercambio de información, las TIC han sido incorporadas en áreas tan disímiles como: ingeniería, matemáticas, ciencias básicas, medicina y administración, por mencionar algunas (González y Blanco, 2011; Piratoba y Rojas, 2014; Parra et al., 2014; Niebles et al., 2016).

Las TIC son un gran recurso que facilita la comunicación sin afrontar las barreras de tiempo y lugar, lo cual ha permitido establecer nuevas formas de interacción entre los actores del proceso enseñanza aprendizaje (Torres y Duarte, 2016; Parada y Suárez, 2014). Adicionalmente, se realizan grandes esfuerzos para desarrollar y evaluar las competencias informacionales en los estudiantes de los diversos niveles educativos, para garantizar una adecuada formación en cuanto a la búsqueda, selección, organización y uso de la información (Gutiérrez et al., 2017, Luis A Mesa Mesa, Nelson Barrera Lombana, 2013).

\subsubsection{Operadores mecánicos}

Gómez et al., (2000) definen los operadores como "sistemas mecánicos que transmiten el movimiento" (p.63). Entre los principales operadores mecánicos se encuentran la rueda, polea, rueda dentada, rueda excéntrica, manivela, cigüeñal, palanca, cremallera, leva, biela, émbolo y tornillo sin fin.

En otras palabras, los operadores mecánicos son objetos, piezas o combinación de ellas que al aplicarles, de forma adecuada, una fuerza mecánica la transforman en movimiento (Marín y Segura, 1998). Su importancia radica en que los operadores mecánicos forman parte de la mayoría de los artefactos que nos rodean, haciendo de su estudio una necesidad para el fomento de la creatividad de los estudiantes (Valdelamar et al., 2015, GuzmánLuna J et al., 2014).

\subsubsection{Objeto de aprendizaje y SCORM}

Astudillo (2011) define Objeto de aprendizaje como "una unidad didáctica digital diseñada para alcanzar un objetivo de aprendizaje simple, y para ser reutilizada en diferentes Entornos Virtuales de Enseñanza y Aprendizaje, y en distintos contextos de aprendizaje. Debe contar, además, con metadatos que propicien su localización, y permitan abordar su contextualización” (p.34).
Los paquetes SCORM permiten integrar contenidos digitales en ambientes de aprendizaje, ya que propician la interoperabilidad entre las diferentes herramientas disponibles en la web para la creación y publicación de los mismos. Sus siglas provienen de Sharable Content Object Reference Model, SCORM. Son un Modelo de referencia de objetos de contenido compartible; es decir, una serie de estándares que regulan el proceso de creación de Objetos de Aprendizaje (OA) en cuanto a la estructura, la ejecución de la secuencia de contenidos, el etiquetado, el empaquetado, la compatibilidad y la comunicación con el ambiente de aprendizaje donde va a ser ejecutado (Del Blanco et al., 2011).

Lo que hace tan útiles este conjunto de estándares es que, además de los metadatos, los métodos para empaquetar los contenidos en formato ZIP, incluyen también los protocolos de comunicación entre el OA creado y el sistema de gestión de aprendizaje, LMS por sus siglas en inglés, donde va a ser publicado. Astudillo et al., (2012), destacan que entre los principales programas que permiten la generación de paquetes SCORM, que son herramientas de autor y que no requieren conocimientos avanzados de programación, se encuentran: RELOAD Editor, Exe Learning, Xml SCORM Studio, Xerte y Ardora, entre otros.

\subsection{Metodología}

La propuesta corresponde a una investigación de tipo cuasi-experimental, de la cual Salkind (1999) indica que se diferencia de la experimental en que el investigador no tiene el control total sobre la forma como se crean los grupos o los miembros que pertenecen a cada uno, y este tipo de asignación sucede con anterioridad a la iniciación de la investigación. Campbell y Stanley (1966) citados por González et al., (2011) afirman que: "cuando los grupos están previamente establecidos no se puede respetar la aleatorización” (p.687).

El proyecto de investigación se desarrolla en el Colegio Boyacá de la ciudad de Duitama, el cual atiende a estudiantes de estratos 1, 2 y 3 de niveles de preescolar, básica primaria, secundaria y media del Barrio Boyacá y sectores aledaños. La población objeto de estudio corresponde al grado séptimo, nivel en el cual se trabaja la temática de operadores mecánicos, y para el cual se desarrolla el ambiente de aprendizaje aquí propuesto. 


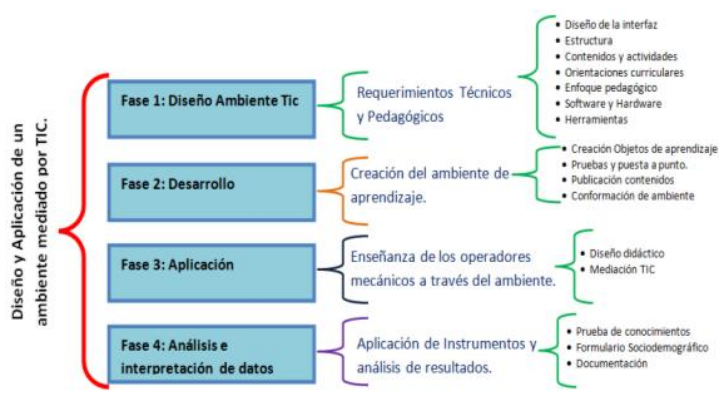

Fig. 1. Esquema de la metodología del proyecto.

La figura 1 presenta el esquema de la metodología propuesta para el diseño y aplicación de un ambiente educativo mediado por TIC; en él se destacan cuatro fases, a saber: la primera corresponde al diseño, en la cual se analizarán los requerimientos técnicos y pedagógicos que se deben tener en cuenta para el desarrollo del Ambiente Virtual de Aprendizaje, AVA, como: el diseño de la interfaz, estructura, contenidos y actividades de acuerdo al modelo constructivista con enfoque significativo que plantea el Proyecto Educativo Institucional, PEI, del Colegio Boyacá y las orientaciones curriculares que plantea el MEN para el Área de Tecnología e Informática.

La segunda fase corresponde al desarrollo del Ambiente Virtual de Aprendizaje, AVA, según la herramienta elegida y el diseño previamente realizado. La tercera fase es de Aplicación donde se implementará la enseñanza de los operadores mecánicos a través del AVA, en el grupo experimental, para evaluar su pertinencia y el grado de aceptación de los estudiantes. La cuarta fase corresponde al análisis e interpretación de la información recolectada para determinar la efectividad del AVA desarrollado, en el proceso enseñanza aprendizaje, de la temática de operadores mecánicos.

En este documento se reportan resultados de las dos primeras fases, que corresponden al diseño y creación del ambiente de aprendizaje.

\section{RESULTADOS Y DISCUSIÓN}

A continuación se presenta como resultado el AVA integrado en la plataforma Moodle donde se incorporan todos los contenidos, actividades y evaluación a través de las herramientas que la misma ofrece. Una parte fundamental del ambiente son los objetos de aprendizaje de los operadores mecánicos, que se desarrollaron en la herramienta de autor ExeLearning y exportaron a Moodle como paquetes SCORM.

\subsection{Diseño del ambiente de Aprendizaje}

El diseño del ambiente de aprendizaje se basa en dos aspectos fundamentales:

\subsubsection{Pedagógicos}

En este aspecto se resalta como objetivo de aprendizaje: Reconocer el concepto, estructura, función y aplicación de los operadores mecánicos. Los contenidos del ambiente son:

1. Los operadores Mecánicos

2. Principales operadores Mecánicos entre los que se encuentran la rueda, polea, rueda dentada, rueda excéntrica, manivela, cigüeñal, palanca, cremallera, leva, biela, émbolo y tornillo sin fin.

3. Concepto, partes, funcionamiento y utilidad de cada uno de los Operadores mecánicos.

Para lograr el objetivo propuesto se plantean las siguientes actividades dentro del ambiente: foro, exploración del contenido a través del paquete SCORM, actividades Interactivas, tareas de observación e investigación, chat, cuestionarios e interacción Estudiante-Docente.

Se utiliza la plataforma de aprendizaje Moodle porque su enfoque constructivista va acorde con el modelo pedagógico del Colegio Boyacá; el estudiante construye su conocimiento: explorando los contenidos, realizando búsquedas, interactuando con los compañeros y docentes (Torres, 2012; Garcés y Ruíz, 2016). Además, el estudiante le da significado a sus conocimientos, aplicando lo aprendido en la construcción de proyectos tecnológicos que luego serán presentados a la comunidad educativa en la muestra Institucional de Tecnología e Informática.

El papel que desempeña el docente del área será el de mediador del conocimiento y del uso de las herramientas TIC. Cabe destacar que su presencia es indispensable en razón a que el tema debe ser desarrollado en las dos horas semanales que tiene asignadas el área de Tecnología e Informática, haciendo uso de los recursos tecnológicos de la sala de informática del colegio, porque no todos los estudiantes cuentan con computador y acceso a internet en sus hogares. El ambiente de aprendizaje planteado se muestra en la figura 2. 


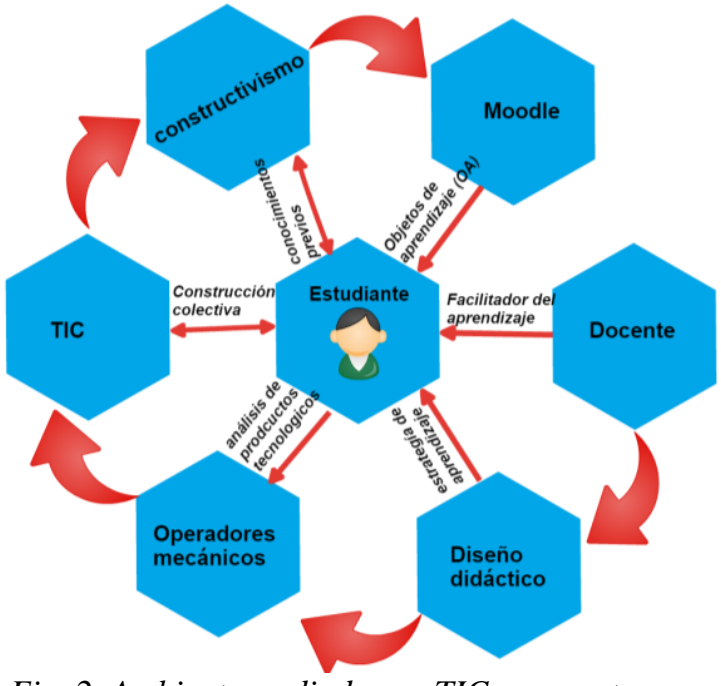

Fig. 2. Ambiente mediado por TIC propuesto para la enseñanza de operadores mecánicos.

El ambiente de aprendizaje que se propone (Figura 2) tiene como eje central al estudiante,de quien se espera se apropie de la temática de operadores mecánicos. En la plataforma Moodle se integran los OA, y se proponen actividades como: tareas, cuestionarios, foros y chats, entre otras, que buscan la construcción de conocimiento para su posterior aplicación en proyectos tecnológicos.

El rol del docente es el de facilitador del proceso formativo mediante la aplicación del diseño didáctico, en el cual se plantea la importancia del análisis de recursos tecnológicos como estrategia de aprendizaje. Es decir, el docente es el encargado de fomentar la participación, intercambio de saberes, procesos de interacción, conceptualización y comunicación de los estudiantes para lograr una construcción colectiva del conocimiento (Boude, 2013).

\subsubsection{Tecnológicos}

El Colegio Boyacá de Duitama cuenta con dos salas de informática, cada una con cuarenta computadores, tablero interactivo y conectividad a internet, la cual se administra por medio de un servidor propiedad de la Institución. Este servidor estaba subutilizado ya que solo se empleaba para controlar los equipos que se podían conectar a la red; se buscó dar uso a estos recursos, remediando en parte la falta de un espacio para trabajar las temáticas relacionadas con tecnología.

La solución implicó la instalación de un software que permitiera gestionar contenidos de aprendizaje, que no implicara una gran inversión económica, por lo que se instaló la plataforma de software libre Moodle; en ella se creó un ambiente de aprendizaje para la temática de Operadores Mecánicos. Esta plataforma permite incorporar y visualizar objetos de aprendizaje realizados en otras aplicaciones que estén empaquetados bajo los estándares SCORM.

Los operadores mecánicos son un tema fundamental en el área de Tecnología e Informática, dada la gran cantidad de aplicaciones en el ámbito educativo e industrial, pues de ellos depende la funcionalidad de aquellas máquinas basadas en el movimiento de sus partes, como: posicionadores, bandas transportadoras, mecanismos para el ajuste de piezas, instrumentos de medida y robots para el desplazamiento de objetos, por mencionar algunas (León y Torres, 2016; Cárdenas y Prieto, 2015; Velazco y Pinto, 2012; Altamirano et al., 2017; Cerón et al., 2013). Sin embargo, luego de efectuar la búsqueda respectiva, no se ha encontrado un objeto de aprendizaje que reúna la mayoría de los operadores mecánicos, describiendo su concepto, partes, utilidad y la aplicación que se les puede dar en la solución de problemas del entorno. Es importante mencionar que en la web se encuentra una gran cantidad de contenido pero de forma individual de cada operador mecánico, cuando lo que se requiere es un objeto de aprendizaje que la gran mayoría.

De acuerdo con lo anterior, se vió la necesidad de crear los objetos de aprendizaje que pudieran ser integrados en la plataforma moodle y que no requirieran mucho tiempo en su elaboración. Para ello se buscaron herramientas gratuitas que permitieran publicar o exportar los OA como paquetes SCORM, estándar compatible con Moodle. La búsqueda permitió identificar a Exe Learning, un software de autor fácil de usar y que permite publicar bajo los estándares requeridos. Adicionalmente, Astudillo y Willging (2012), en su estudio titulado "Análisis de compatibilidad entre Objetos de Aprendizaje basados en SCORM y LMS de Código Abierto", concluyen que "los OA SCORM diseñados con eXe Learning y CourseLab fueron los que mejor se adaptaron al trabajo sobre los LMS analizados"; debido a ello se decidió tomar en cuenta esta recomendación.

El ambiente de aprendizaje está conformado por tres OA desarrollados en el software de autor ExeLearning, utilizando como plantilla el estilo Kids, pensado desde su creación para niños por sus letras grandes, efectos de javascript y con íconos para atraer su atención (Exelearning, sf). Los OA se encuentran publicados en la plataforma como paquetes SCORM e integrados con otras herramientas, como: foros, chat, tareas y cuestionarios. 
Cada OA está conformado por cuatro operadores mecánicos y contiene un menú de navegación que consta de cuatro opciones: la primera es preconceptos, la cual vincula y despliega una página donde aparecen conceptos fundamentales para una mejor comprensión de los operadores mecánicos, como son: máquina, operador y tipos de movimiento. La segunda opción es operadores mecánicos, que además de contener el concepto e imágenes de los mismos, despliega un submenú con el nombre de cada uno de ellos. La tercera opción corresponde a practiquemos, que ofrece actividades interactivas acerca de los cuatro operadores vistos en cada OA; y la cuarta opción referencias Bibliográficas, donde se presentan documentos que permiten ampliar los conceptos bajo estudio.

Los doce operadores mecánicos fueron distribuidos en tres objetos de aprendizaje. El primer OA conformado por: rueda, polea, rueda dentada y rueda excéntrica; El segundo OA: Leva, manivela, cigüeñal y biela; El tercer OA: Palanca, émbolo, cremallera y tornillo sin fín.

Cada Operador mecánico despliega un sistema de 5 pestañas, etiquetadas en forma de pregunta, la primera de ellas es ¿Qué es?, presenta la definición del operador y una animación de su funcionamiento; la segunda ¿Cuáles son sus partes?, presenta un texto que describe cada una de las partes que componen el operador mecánico y una imagen que ilustra su ubicación; la tercera $i$ cómo funciona?, muestra al lado izquierdo una descripción del funcionamiento del operador mecánico y al lado derecho un video demostrativo del portal Youtube embebido, lo que indica que no direcciona al portal sino que se reproduce en el mismo OA; la cuarta responde a ¿cuál es su utilidad?, para ello aparecen varias imágenes de máquinas que están compuestas por el operador mecánico; al pasar el mouse sobre ellas, muestra por medio de una ventana emergente, la función que desempeña y en que otros artefactos está presente y la quinta pestaña corresponde a ¿practicamos?, que son actividades interactivas realizadas en el portal educativo Educaplay y embebidas en el OA acerca del operador mecánico correspondiente, para que el estudiante además de autoevaluarse pueda construir conocimiento por medio de la ejercitación.

\subsection{Diseño didáctico propuesto}

El diseño didáctico propuesto para la aplicación del ambiente de aprendizaje mediado por TIC (Figura
3) considera como estrategia de aprendizaje el análisis de productos tecnológicos para desarrollar la temática de operadores mecánicos, utilizando como herramienta mediadora la plataforma Moodle.

En el diseño propuesto se utiliza como estrategia de aprendizaje el análisis de productos tecnológicos, el cuál puede ser: morfológico, funcional, estructural funcional, tecnológico, económico, comparativo, relacional, ambiental, reconstrucción del surgimiento y la evolución histórica del producto (Balagué et al., 2012). En este caso se analizan los operadores mecánicos desde los puntos de vista morfológico (forma), estructural (elementos que lo componen), y funcional (función), con el fin de que el estudiante comprenda cómo utilizarlos para resolver problemas de su entorno.

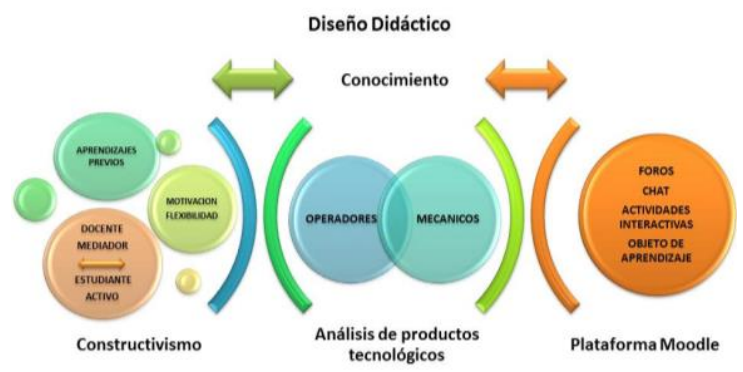

Fig. 3. Diseño didáctico planteado

En cuanto a la mediación con herramientas TIC, como primera actividad, se tiene un foro de presentación donde se busca indagar qué conocimientos previos posee el estudiante acerca de los operadores mecánicos. Luego aparecen los OA descritos en la sección anterior, de modo que el estudiante interactúe con contenidos que le permitan analizar qué son, cómo están compuestos, qué función cumplen y cómo aplicar los operadores mecánicos. Finalmente, el estudiante tendrá como actividad observar y describir cuales de ellos están presentes en los artefactos que usa a diario. El estudiante construye conocimiento a través de las diferentes actividades que desarrolla, con la investigación, con la exploración del objeto de aprendizaje, en la interacción con los compañeros y con el docente, quien desempeña el rol de mediador del proceso formativo y del uso de las herramientas TIC.

El funcionamiento del ambiente de aprendizaje se valoró en una prueba piloto con un grupo de estudiantes de grado Séptimo, que no hacen parte de la población objeto de estudio, con el fin de evaluar su funcionalidad, identificar posibles 
errores y observar si la interfaz facilitaba la navegación de los usuarios. Se evidenció gran aceptación del ambiente debido a: su fácil manejo, todos los enlaces funcionaron correctamente, presenta una interfaz muy intuitiva, las actividades importadas de otras aplicaciones no presentaron problemas en el cargue; además, los colores y el diseño de los OA fueron adecuados para la edad de los educandos. La única modificación se debió a que los estudiantes tuvieron inconvenientes para enviar archivos a la plataforma, pues el peso máximo permitido era $1 \mathrm{MB}$, por lo cuál se amplió este valor en la configuración.

\section{CONCLUSIONES}

En este trabajo se diseñó y desarrolló un ambiente de aprendizaje mediado por TIC, alojado en la plataforma Moodle, utilizando Objetos de Aprendizaje realizados en Exe Learning y publicados bajo el estándar SCORM. En los objetos de aprendizaje se presenta el concepto, estructura, funcionamiento y utilidad de los operadores mecánicos, temática importante en el área de tecnología e informática de la educación básica.

Las pruebas del ambiente permitieron establecer que los objetos de aprendizaje se integran con éxito a la plataforma Moodle, gracias a su compatibilidad con paquetes SCORM. Estos estándares facilitan la publicación en las plataformas administradoras de contenido, LMS por sus siglas en inglés, de recursos elaborados en otras herramientas.

El diseño didáctico se planteó con un enfoque constructivista, con el docente como orientador y motivador del proceso de aprendizaje aplicando la estrategia de análisis de productos tecnológicos en el desarrollo de la temática de operadores mecánicos. La herramienta mediadora seleccionada fue la plataforma Moodle, la cual permite actividades de tipo socio constructivista como: Foros, Chat, Actividades interactivas, integración de Objetos de Aprendizaje y la interacción entre el estudiante con sus compañeros y con el docente, que en este caso se presenta como mediador del conocimiento y el uso de las herramientas TIC. El trabajo futuro será el desarrollo del tema operadores mecánicos con los grupos experimental y control, usando con el primero el ambiente de aprendizaje mediado por TIC, para luego aplicar una prueba de conocimientos en las mismas condiciones a los dos grupos, que permita valorar el impacto del ambiente propuesto en el aprendizaje de los estudiantes.

\section{REFERENCIAS}

Altamirano S, E., Vallejo V, G., y Cruz H, J. (2017). Monitoreo volcánico usando plataformas Arduino y Simulink. Revista de Investigación, Desarrollo e Innovación, 7(2), 317-329. doi:https://doi.org/10.19053/20278306.v7. n2.2017.6073.

Algemiro A. Ávila G, Elkin G. Flórez Serrano, Oscar Gualdrón G (2012). Diseño e implementación de un curso interactivo multimedia para el aprendizaje de los procesos CAM en un centro de mecanizado Leadwell V30. Revista Colombiana de Tecnologías de Avanzada ISSN: 1692-7257 Volumen 2 - Número 20.

Angarita V, M. A., Fernández M, F. H., y Duarte, J. E. (2014). La didáctica y su relación con el diseño de ambientes de aprendizaje: una mirada desde la enseñanza de la evolución de la tecnología. Revista de Investigación, Desarrollo e Innovación, 5 (1), 46-55. Doi: https://doi.org/10.19053/20278306.3138

Angarita V, M., Fernández M, F., y Duarte, J. (2016). Formación de ingenieros interdisciplinarios a través de una metodología activa con temáticas integradoras. Saber, Ciencia Y Libertad, 11(2), 177-187. doi:http://dx.doi.org/10.22525/sabcliber20 $16 \mathrm{v} 11 \mathrm{n} 2.202$

Astudillo, G. J. (2011). Análisis del estado del arte de los objetos de aprendizaje. Revisión de su definición y sus posibilidades. La plata: Universidad Nacional de La Plata.

Astudillo, G. J., Sanz, C., y Willging, P. A. (2012). Análisis de Compatibilidad entre Objetos de Aprendizaje basados en SCORM y LMS de Código Abierto. Conferencias LACLO, 3 (1).

Balagué , L. J., Pastorino , G. N., Diosma, G., Martínez A, V., Fermoselle, G., Guaymasí, D., . . Balatti, P. A. (2012). Aplicación de la metodología "análisis de producto Tecnológico" en la enseñanza de microbiología agrícola Empleando inoculantes microbianos. En IV Congreso Nacional y III Congreso Internacional de Enseñanza de las Ciencias Agropecuarias.

Boude-Figueredo, O. R. (2013). Tecnologías emergentes en la educación: Una experiencia de formación de docentes que fomenta el 
diseño de ambientes de aprendizaje. Educ. Soc. Campinas, 531-548. Recuperado de http://www.cedes.unicamp.br

Boude F, O., y Medina R, A. (2011). Desarrollo de competencias a través de un ambiente de aprendizaje mediado por TIC en educación superior. Educación Médica Superior, 25 (3), 301-311.

Cárdenas, J. A., y Prieto O, F. A. (2015). Diseño de un algoritmo de corrección automática de posición para el proceso de perforado PCB, empleando técnicas de visión artificial. Revista de Investigación, Desarrollo e Innovación, 5 (2), 107-118. doi: $10.19053 / 20278306.3720$

Castro G, J. C., Pinto S, M. L., y Amaya Q, M. F. (2014). Diseño y construcción de una Bobina Tesla de $1680 \mathrm{~W}$, para la enseñanza de conceptos básicos en sistemas eléctricos de potencia. Revista de Investigación, Desarrollo e Innovación, 5(1), 66-74. Doi: https://doi.org/10.19053/20278306.3142

Cerón C, A., Salazar J, A. E., y Prieto O, F. A. (2013). Reconocimiento de rostros y gestos faciales mediante un análisis de relevancia con imágenes 3D. Revista de Investigación, Desarrollo e Innovación, 4 (1), 7-20. doi: 10.19053/20278306.2563

Clayton J Torres, John F Archila, Mario L Tronco, Marcelo Becker, Arthur J Viera Porto, Alexander J Tiberti (2013). ESTUDIO CINEMÁTICO DE UNA PLATAFORMA ROBÓTICA PARA AGRICULTURA. Revista Colombiana de Tecnologías de Avanzada ISSN: 1692-7257 - Volumen 2 Número 22.

Del Blanco A, A., Torrente, J., Martinez O, I., y Fernandez M, B. (2011). Análisis del Uso del Estándar SCORM para la Integración de Juegos Educativos. IEEE-RITA, 6 (3), 118127.

Exelearning (sf). ExeLearning 2.1. Tutorial Manual. Recuperado de: http://exelearning.net/html_manual/exe 20/descarga_de_las_fuentes.html.

Garcés P, M., Ruiz C, R. y Martínez A, D. (2014). Transformación pedagógica mediada por tecnologías de la información y la comunicación (TIC). Saber, Ciencia y Libertad, 9 (2), 217-228. Recuperado de: https://dialnet.unirioja.es/descarga/articul o/5104968.pdf

Garcés P, M., y Ruiz C, R. (2016). Integración pedagógica de la tecnología informática en instituciones educativas oficiales de Cartagena de indias (Colombia). Saber,
Ciencia Y Libertad, 11(1), 175-186. doi:http://dx.doi.org/10.22525/sabcliber20 $16 \mathrm{v} 11 \mathrm{n} 1.184$.

Góméz O, L. A., Silva R, F., Jiménez A, J., y Alamaraz M, A. (2000). Educación en Tecnología 1. Madrid: Mc Graw-Hill /Interamericana de España S.A.

González C., Coll, D., Sicilia C, A., y Moreno M, J. A. (2011). Un estudio cuasi-experimental de los efectos del clima motivador tarea en las clases de Educación Física. Revista de educación, 677-700. doi: 10-4438/1988592X-RE-2010-356-056.

González L, J. J., y Blanco A, N. (2011). Estrategia didáctica con mediación de las TIC, propicia significativamente el aprendizaje de la Químmica Orgánica en la educación secundaria. Escenarios, 9 (2), 7-17.

González, D., Estrada, E., y Roldán, J. (2016). Aplicación Android para el estudio de mecanismos planos de cuatro barras. Entre Ciencia e Ingeniería, 10 (20), 41-51. Recuperado de: http://www.scielo.org.co/scielo.php?scrip $\mathrm{t}=$ sci_arttextypid=S1909-

$83672016000200007 y \operatorname{lng}=$ esytlng=es.

Gutiérrez C, J. J., Cabero A, J., y Estrada V, L. I. (2017). Diseño y validación de un instrumento de evaluación de la competencia digital del estudiante universitario. Revista Espacios, 38 (10), 16. Recuperado de: http://www.revistaespacios.com/a17v38n 10/17381018.html.

Jaramillo M, P., Ordóñez O, C. L., Castellanos G, S. E., y Castañeda B, C. P. (2005). nformática, todo un reto: ambientes de aprendizaje en el aula de informática : ¿fomentan el manejo de información? Bogotá, Colombia: Uniandes.

Jaime Guzmán-Luna, Ingrid-Durley Torres, Juan Felipe Alvarez (2014). PROPUESTA DE UN GENERADOR DE APLICACIONES EDUCATIVAS BASADAS EN TELEVISIÓN DIGITAL USANDO ARQUITECTURA DE CÓMPUTO EN LA NUBE. Revista Colombiana de Tecnologías de Avanzada ISSN: 1692-7257 - Volumen 2 Número 24.

León M, J. X., y Torres B, E. A. (2016). Herramienta para el diseño de sistemas de posicionamiento tridimensional usados en fabricación digital. Revista de Investigación, Desarrollo e Innovación, 6 (2), 155-167. doi: http://doi.org/10.19053/20278306.4603

Luis A Mesa Mesa, Nelson Barrera Lombana (2013). La robótica educativa como 
instrumento didáctico alternativo en educación básica. Revista Colombiana de Tecnologías de Avanzada ISSN: 1692-7257 Volumen 2 - Número 22.

Marín M, N., y Segura G, L. M. (1998). Construcción de un cuestionario sobre operadores mecánicos. En S. García Barros, y C. Martínez Losada, La didáctica de las ciencias: tendencias actuales (págs. 395-405). Universidade da Coruña.

Ministerio de Educación Nacional, MEN. (2008). Ser competente en tecnología. Bogotá, Colombia: Imprenta Nacional.

Montes M, A. J. (2013). La educación básica en Colombia: una mirada a las políticas educativas. Saber, Ciencia y Libertad, 8 (2), 141-155. Recuperado de: http://www.sabercienciaylibertad.com/ojs /index.php/scyl/article/view/106.

Niebles N, W., Hernández P, H., y Cardona A, D. (2016). Gestión tecnológica del conocimiento: herramienta moderna para la gerencia de instituciones educativas. Revista de Investigación, Desarrollo e Innovación, 7(1), 25-36. doi:https://doi.org/10.19053/20278306.v7. $\mathrm{n} 1.2016 .5633$.

Niño V, J. A., Martínez D, L. Y., y Fernández M, F. H. (2016). Mano robótica como alternativa para la enseñanza de conceptos de programación en Arduino. Revista Colombiana de Tecnologías de Avanzada, 2 (28), 132-139.

Núñez P, B., González C, J., y Viloria M, P. (2014). Carril de aire con sensor óptico, utilizado en la realización de experiencias en cinemática en los laboratorios de física mecánica. Ingeniare, 9 (16), 17-24.

Parada H, A., y Suárez A, Z. E. (2014). Influencia de las Tecnologías de la Información y la Comunicación en la apropiación de conceptos de electrónica análoga, en estudiantes de grado séptimo de educación básica. Revista de Investigación, Desarrollo e Innovación, 5(1), 20-31. Doi: https://doi.org/10.19053/20278306.3137.

Parra L, L. F., Duarte, J. E., y Fernández M, F. H. (2014). Propuesta didáctica para la enseñanza de circuitos eléctricos básicos. Revista de Investigación, Desarrollo e Innovación, 4 (2), 138-147.

Doi: http://doi.org/10.19053/20278306.2891.

Piratoba G, R. P., y Rojas M, C. E. (2014). Cambios en las concepciones iniciales e inducidas sobre la naturaleza de las matemáticas y su didáctica, en estudiantes de un programa de Licenciatura en Matemáticas y Estadística. Revista de Investigación, Desarrollo e Innovación, 5(1), 32-45. Doi: https://doi.org/10.19053/20278306.3140.

Rodríguez C, R. (2016). Aprendizaje de conceptos químicos: una visión desde los trabajos prácticos y los estilos de aprendizaje. Revista de Investigación, Desarrollo e Innovación, $7(1)$, 63-76. doi:http://dx.doi.org/10.19053/20278306. v7.n1.2016.4403.

Salkind, N. J. (1999). Métodos de investigación. Pearson Educación.

Solbes, J., y Vilches, A. (1992). El modelo constructivista y las relaciones CTS. Enseñanza de las ciencias, 181-186.

Torres O, J. A. (2012). Incidencia de Moodle en las prácticas pedagógicas en modalidad educativa B-Learning. Revista de Investigación, Desarrollo e Innovación, 2 (2), 39-48. Recuperado http://revistas.uptc.edu.co/index.php/inve stigacion_duitama/article/view/1315.

Torres O, J. A., y Duarte, J. E. (2016). Los procesos pedagógicos administrativos y los aspectos socio-culturales de inclusión y tecno-pedagogía a través de las tendencias pedagógicas en educación a distancia y virtual. Revista de Investigación, Desarrollo e Innovación, 6(2), 179-190. doi: http://doi.org/10.19053/20278306.4606.

Valdelamar Z, J. A., Ramírez C, Y. L., Rodríguez R, P. D., y Morales R, M. E. (2015). Capacidad innovadora: cómo fomentarla, según docentes de Ciencias Económicas e Ingeniería de la UMNG. Revista de Investigación, Desarrollo e Innovación, 6(1), 7-14.

doi: http://doi.org/10.19053/20278306.3454.

Velazco C, D. F., y Pinto S, M. L. (2012). Caracterización del huevo de gallina para el diseño de un sistema automático de clasificación. Revista de Investigación, Desarrollo e Innovación, 3 (1), 33-43. 